(C) [2008] IEEE. Reprinted, with permission, from [Thanh H. Nguyen, Jordan S. Nguyen and Hung T. Nguyen, Bayesian recursive algorithm for width estimation of freespace for a power wheelchair using stereoscopic cameras, Engineering in Medicine and Biology Society, 2008. EMBS 2008. 30th Annual International Conference of the IEEE, 20-25 Aug. 2008]. This material is posted here with permission of the IEEE. Such ermission of the IEEE does not in any way imply IEEE endorsement of any of the University of Technology, Sydney's products or services. Internal or personal use of this material is permitted. However, permission to reprint/republish this material for advertising or promotional purposes or for creating new collective works for resale or redistribution must be obtained from the IEEE by writing to pubs-permissions@ieee.org. By choosing to view this document, you agree to all provisions of the copyright laws protecting it 


\title{
Bayesian Recursive Algorithm for Width Estimation of Freespace for a Power Wheelchair Using Stereoscopic Cameras
}

\author{
Thanh H. Nguyen, Jordan S. Nguyen, Hung T. Nguyen, Senior Member, IEEE
}

\begin{abstract}
This paper is concerned with the estimation of freespace based on a Bayesian recursive (BR) algorithm for an autonomous wheelchair using stereoscopic cameras by severely disabled people. A stereo disparity map processed from both the left and right camera images is constructed to generate a 3D point map through a geometric projection algorithm. This is then converted to a 2D distance map for the purpose of freespace estimation. The width of freespace is estimated using a BR algorithm based on uncertainty information and control data. Given the probabilities of this width computed, a possible movement decision is then made for the mobile wheelchair. Experimental results obtained in an indoor environment show the effectiveness of this estimation algorithm.
\end{abstract}

\section{INTRODUCTION}

$\mathrm{P}$ ower wheelchairs are necessary to provide mobility assistance for severely disabled people. However a conventional wheelchair in many cases is not sufficient to assist in their mobility. For this reason, certain wheelchairs are fitted with additional equipment, such as sensors, and given new, generally autonomous, capabilities such that they can be classified as smart wheelchairs, in order to enhance the independence and mobility of severely disabled people. In recent decades many significant algorithms, which utilise the additional equipment, have been developed to create these smart wheelchairs [1].

Cameras are one type of sensory equipment that can be mounted on robots and wheelchairs to obtain real-time information from the environment. This information is analysed and processed by advanced algorithms to produce necessary data and signals for the operation of the associated robots and smart wheelchairs [2,3]. In particular, the left and right images acquired from stereoscopic cameras are matched to generate a disparity map using a Sum of Absolute Differences (SAD) algorithm [4]. This disparity map is used to produce a $3 \mathrm{D}$ point map, which is then applied a geometric projection algorithm to convert it to a 2D distance map [5-7]. These 2D distance maps can allow detection of obstacles and freespace distances necessary in the operation of robots and smart wheelchairs.

This work was supported in part by Australian Research Council under Discovery Grant DP0666942 and LIEF Grant LE0668541.

Thanh H. Nguyen is with Faculty of Engineering, University of Technology, Sydney, Broadway, NSW 2007, Australia (phone: +612-95142451; fax: +61 29514 2868; e-mail: thnguyen@eng.uts.edu.au).

Jordan S. Nguyen is with Faculty of Engineering, University of Technology, Sydney, Broadway, NSW 2007, Australia (e-mail: Jordan.Nguyen@student.uts.edu.au).

Hung T. Nguyen is with Faculty of Engineering, University of Technology, Sydney, Broadway, NSW 2007, Australia (e-mail: Hung.Nguyen@uts.edu.au).
The Bayesian method is useful in detecting freespace from sensor information for robots. For example, in a recent application, an autonomous exploration strategy for freespace detection based on mapping uses the Bayesian update theory [8]. A Bayesian filter algorithm with statistical tool is also applied to estimate locations based on uncertainty information from sensors. This Bayesian filter algorithm uses the Markov assumption and recursive process to compute the belief probability [9].

The BR algorithm is applied to estimate the width of freespace in a 2D distance map using measurements and control data from the wheelchair system. Firstly the previous probability is computed based on the prior probability and the control data. The second step of this algorithm is that the posterior probability using Bayesian rules is carried out based on the previous probability along with the measurement update. The process is recursively iterated in the next time step to choose the high belief probability. The average of the probabilities is to make a decision for the mobile wheelchair.

This paper is organised as follows. In Section 2, the problem of stereoscopic vision is introduced. In Section 3, a BR algorithm using Bayesian rules, control data and measured information is employed to estimate the width of freespace. Section 4 shows the experimental result, in which the width of freespace in a 2D distance map is estimated to make the decision. Finally Section 5 concludes the paper.

\section{STEREOSCOPIC VISION}

\section{A. Stereo Disparity}

The purpose of stereo vision is to perform range measurements based on the left and right images obtained from stereoscopic cameras. Basically, a correlation algorithm of the Sum of Absolution Differences (SAD) is implemented to establish the correspondence between image features in different views of the scene and then calculate the relative displacement between feature coordinates in each image to produce a disparity [5].

$$
S A D=\min _{d=d_{\min }}^{d_{\max }} \sum_{i=-M}^{+M} \sum_{j=-M}^{+M}\left|I_{R}(x+i, y+j)-I_{L}(x+i+d, y+j)\right|
$$

where the centre of a window of size $(2 M+1) \times(2 M+1)$ at the coordinates of the matching pixels $(i, j),(x, y)$, are pixel coordinates in one image. $I_{L}$ and $I_{R}$ are the intensity functions of the right and left images, and $d_{\min }, I_{\max }$ are the minimum and maximum disparities. The disparity $d_{\min }$ of zero pixels often corresponds to an infinitely far away object and $d_{\max }$ denotes the closest position of an object. 
B. Computation of a $2 D$ Distance Map

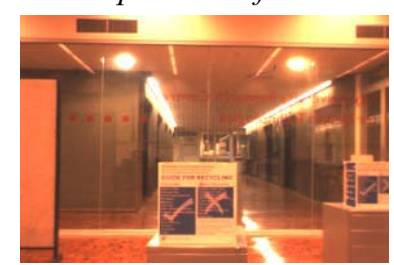

Fig. 1: Left image

$\mathrm{A} Z(\mathrm{~m})$

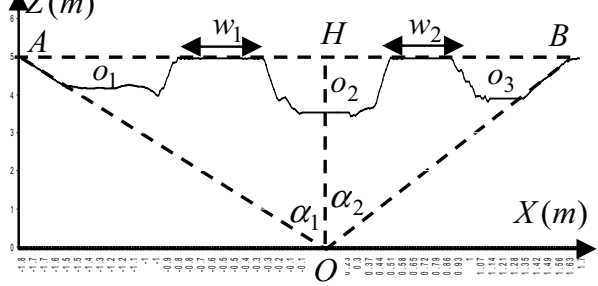

Fig. 3: 2D distance map

In this project, a Point Grey Research 'Bumblebee 2' stereoscopic camera system used to capture the left and right images as shown in Fig. 1 and Fig. 2 has two cameras which act like two "eyes". This camera system has a limited field of view, with the total range denoted here as $\alpha$. This vision system produces a $\alpha_{1}$ maximum angle to the left and a $\alpha_{2}$ maximum angle to the right of the wheelchair due to the angle of the cameras system relative to the wheelchair. It means that the camera system can just see freespaces $w_{1}, w_{2}$ and obstacles $o_{1}, O_{2}$ and $o_{3}$ in the field of view $(A O B)$ as shown in Fig. 3, in which $Z$ is distance-axis, $X$ is horizontalaxis and $O H=Z_{\max }$ is the maximum visible distance of the cameras. The maximum range of $Z$-axis is $5000 \mathrm{~mm}$, the 0.01 resolution of $X$-axis is from $-1800 \mathrm{~mm}$ to $1700 \mathrm{~mm}$ and the image resolution in this case is $320 \times 640$ in the $2 \mathrm{D}$ map.

Given the camera calibration, a stereo disparity map is processed to generate a $3 \mathrm{D}$ point cloud image based on subpixel interpolation for more accurate stereo depth extraction. From this $3 \mathrm{D}$ point image, four steps can be performed to convert this to a $2 \mathrm{D}$ distance map using geometric projection as follows:

- Step 1: n 3D points $\left(x_{i}, y_{j}, z_{k}\right)$ are converted to $\mathrm{n} 3 \mathrm{D}$ points $\left(X_{i}, Y_{j}, Z_{k}\right)$ on coordinates $X, Y, Z$.

- Step 2: Assume that a certain value $X_{i}$ on the horizontal $X$-axis corresponds to many points $\left(Y_{j}, Z_{k}\right)$. The minimum distance $Z_{\text {imin }}$, from an object to the wheelchair corresponding to a point $X_{i}$, is chosen.

- Step 3: In order to obtain a 2D distance map $\left(X_{i}, Z_{\text {imin }}\right)$, all minimum distances $Z_{\text {imin }}$ are computed as follows

$$
Z_{i \min }=\min _{j=0}^{k} Z_{i j}
$$

- Step 4: The width of freespace $w$ is detected based on the maximum distance $Z_{\text {imax }}$ as follows

$$
w=\sum Z_{i \max }
$$

\section{FREESPACE ESTIMATION ALGORITHM}

\section{A. Bayesian Recursive Algorithm}

The width of freespace in a 2D distance map is difficult to estimate due to noise information from the camera system.
In order to solve this problem, Bayesian Recursive (BR) algorithm bases on measurements and control data of the wheelchair system to compute the conditional probability. The first step is that the algorithm requires the probability and the control $u(t)$ to compute the probability over the width of freespace $w(t)$ which is a state variable. The prior probability is expressed as follows

$$
P_{p r}(w(t))=\sum_{w(t-1)} P(w(t-1) \mid w(t-1), u(t)) P(w(t-1))
$$

in which $P(w(t-1))$ and $P(w(t) \mid w(t-1), u(t-1))$ are the previous probability and the conditional probability over the width $w(t-1)$.

The next step is that, the probability $P_{p r}\left(w_{t}\right)$ is used along with the conditional probability based on measurements $z_{t}$ to make the posterior probability as follows

$$
P_{p o}(w(t))=\frac{P(z(t) \mid w(t)) P_{p r}(w(t))}{\sum_{w(t)} P(z(t) \mid w(t)) P_{p r}(w(t))}
$$

where Bayes theorem is utilised for the posterior probability $P_{p o}(w(t))$ based on the measurement and $\Sigma P_{p o}(w(t))=1$.

$P_{p o}(w(t))$ will be the previous probability for the next time step to determine the previous probability $P_{p r}(w(t))$ based on the control in Eq. (4). This means that this algorithm is recursively carried out to compute the high belief probability for the dynamic system.

\section{B. Decision Making}

This algorithm is recursively iterated many times to produce the probabilities. The average of the probabilities is computed to make a decision for the wheelchair as follows

$$
p_{a v}(w)=\sum_{i=1}^{n} \frac{P_{p o, n}(w(t))}{n}
$$

where $n$ is the amount of times.

The average probability is then compared to the threshold probability $P_{t h}$ that is carried out many times in the practical operation of the wheelchair. If $P_{a v}(w)$ is greater than or equal to $p_{t h}$, the mobile wheelchair can be decided to move.

\section{EXPERIMENTAL RESULTS}

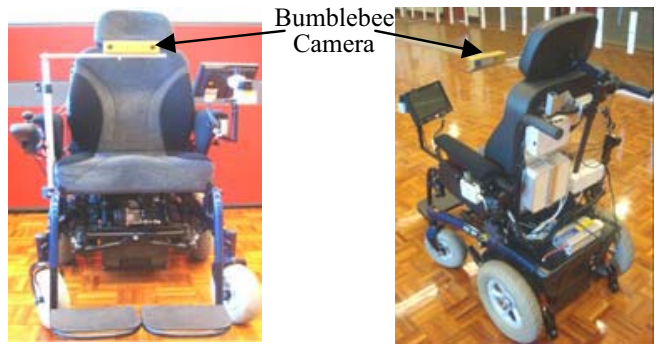

Fig. 4: Power wheelchair

The autonomous wheelchair has been fitted with the 'Bumblebee 2' camera system in the University Research Centre for Health Technologies as shown in Fig. 4.

Consider an actual width of a freespace $w_{a}$ of an entry through a doorway or a corridor which is compared to the safe diameter $d_{s}$ of a wheelchair. If $w$ measured by the camera system is greater than or equal to $d_{s}$, the wheelchair 
can move through this freespace. If $w$ is less than $d_{s}$, the wheelchair can not move through. In practice, $w$ measured by the camera system can be less than, equal to or greater than $w_{a}$. This is very difficult to estimate. To solve this problem, BR algorithm is employed to estimate the width of freespace based on the conditional probabilities, control data and measurements. Two practical experiments implemented to estimate the width of freespace are shown below.

\section{A. Experiment 1}

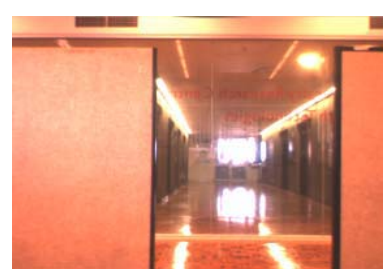

Fig. 5: Left image

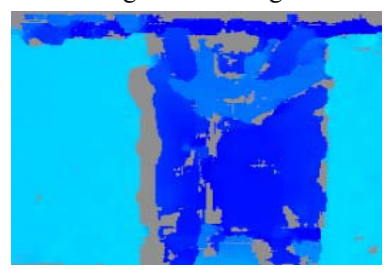

Fig. 7: Stereo disparity map

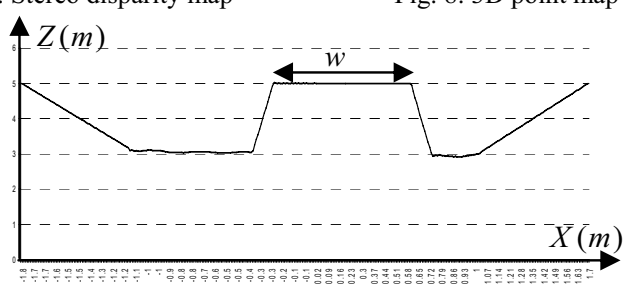

Fig. 9: 2D distance map

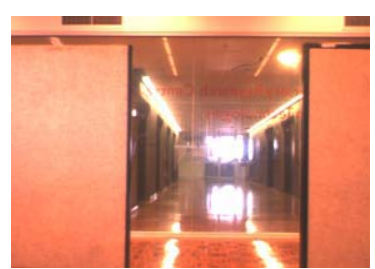

Fig. 6: Right image

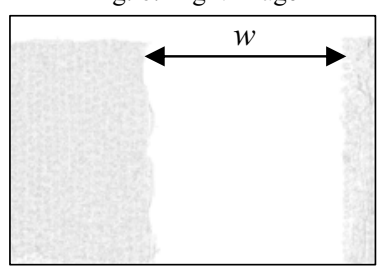

Fig. 8: 3D point map

$$
p(z(t)=O B \mid w(t)=O B)=0.8
$$

in which $z(t)=F S$, if $w(t)$ measured by the camera system is greater than or equal to $1000 \mathrm{~mm}$ and $z(t)=O B$, if $w(t)$ measured by the camera system is less than $1000 \mathrm{~mm}$.

The conditional probabilities depend on the wheelchair control $u(t)=$ move and its previous state as follows

$$
\begin{aligned}
& p(w(t)=F S \mid w(t-1)=F S, u(t)=\text { move })=0.8 \\
& p(w(t)=O B \mid w(t-1)=F S, u(t)=\text { move })=0.2 \\
& p(w(t)=F S \mid w(t-1)=O B, u(t)=\text { move })=0.9 \\
& p(w(t)=O B \mid w(t-1)=O B, u(t)=\text { move })=0.1
\end{aligned}
$$

For similarity, the control case is $u_{t}=s t o p$, the conditional probabilities are determined as follows

$$
\begin{aligned}
& p(w(t)=F S \mid w(t-1)=F S, u(t)=\text { stop })=0.1 \\
& p(w(t)=O B \mid w(t-1)=F S, u(t)=\text { stop })=0.9 \\
& p(w(t)=F S \mid w(t-1)=O B, u(t)=\text { stop })=0.1 \\
& p(w(t)=O B \mid w(t-1)=O B, u(t)=\text { stop })=0.9
\end{aligned}
$$

In practice, relatively the error probabilities can occur as shown in Eqs. (10b), (11b), (12a) and (13a).

Firstly, the wheelchair takes on control action $u(1)=$ stop and the measurement $z(1)=F S$ at time $t_{1}$. From Eq. (4), the probabilities $P_{p r}(w(1))$ using the prior probability are computed for the width $w(1)$ as follows

$$
\begin{aligned}
& P_{p r}(w(1)=F S)=\sum_{w(0)} P(w(1) \mid w(0), u(1)) P(w(0))=0.5(14 \mathrm{a}) \\
& P_{p r}(w(1)=O B)=\sum_{w(0)} P(w(1) \mid w(0), u(1)) P(w(0))=0.5(14 \mathrm{~b})
\end{aligned}
$$

Given the measurement $z(1)=F S$ and $P_{p r}(w(1))$, the posterior probabilities $P_{p o}(w(1))$ using Eq. (5) are determined as follows

$$
\begin{aligned}
& P_{p o}(w(1)=F S)=\frac{P(z(1)=F S \mid w(1),) P_{p r}(w(1))}{\sum_{w(1)} P(z(1)=F S \mid w(1)) P_{p r}(w(1))}=0.778(15 \mathrm{a}) \\
& P_{p o}(w(1)=O B)=\frac{P(z(1)=F S \mid w(1),) P_{p r}(w(1))}{\sum_{w(1)} P(z(1)=F S \mid w(1)) P_{p r}(w(1))}=0.222(15 \mathrm{~b})
\end{aligned}
$$

This BR algorithm is iterated for the next time step corresponding to $u(2)=$ move at time $t_{2}$, we obtain the probabilities using Eq. (4) as follows

$$
\begin{aligned}
& P_{p r}(w(2)=F S)=0.8778 \\
& P_{p r}(w(2)=O B)=0.1222
\end{aligned}
$$

As the measurement is still $z(2)=F S$, we have the posterior probabilities using Eq. (6) as follows

$$
\begin{aligned}
& P_{p o}(w(2)=F S)=0.9617 \\
& P_{p o}(w(2)=O B)=0.0383
\end{aligned}
$$

This process is recursively iterated ten times to produce the probabilities $P_{p o}(w=F S), P_{p o}(w=O B)$ and the average probabilities, $P_{a v}(w=F S), P_{a v}(w=O B)$ as shown in Table 1. If $P_{p o}(w) \geq d_{s}$ then there is a freespace and if $P_{p o}(w)<d_{s}$ there is an obstacle. In particular, the average probability $P_{a v}(w=F S)=0.8821$ is greater than $P_{t h}=0.8$, it means that the
Furthermore, the conditional probabilities corresponding time $t$ are carried out in fact by trial times

$$
\begin{aligned}
& p(z(t)=F S \mid w(t)=F S)=0.7 \\
& p(z(t)=O B \mid w(t)=F S)=0.3 \\
& p(z(t)=F S \mid w(t)=O B)=0.2
\end{aligned}
$$


width $w$ is the freespace and the wheelchair is possibly decided to go through.

Table 1: The estimated values of the width of one freespace

\begin{tabular}{|c|c|c|c|}
\hline Times & $w(t)$ & $P_{p o}(w(t)=F S)$ & $P_{p o}(w(t)=O B)$ \\
\hline 1 & 1020 & 0.7778 & 0.2222 \\
\hline 2 & 1020 & 0.9617 & 0.0383 \\
\hline 3 & 980 & 0.7640 & 0.2360 \\
\hline 4 & 1020 & 0.9613 & 0.0387 \\
\hline 5 & 1020 & 0.9679 & 0.0321 \\
\hline 6 & 1000 & 0.9682 & 0.0318 \\
\hline 7 & 970 & 0.7652 & 0.2348 \\
\hline 8 & 990 & 0.7269 & 0.2731 \\
\hline 9 & 1010 & 0.9600 & 0.0400 \\
\hline 10 & 1020 & 0.9679 & 0.0321 \\
\hline Average probability $P_{a v}$ & $\mathbf{0 . 8 8 2 1}$ & $\mathbf{0 . 1 1 7 9}$ \\
\hline \multicolumn{2}{|r|}{ Variance } & $\mathbf{0 . 0 1 1 5}$ & $\mathbf{0 . 0 1 1 5}$ \\
\hline
\end{tabular}

\section{B. Experiment 2}

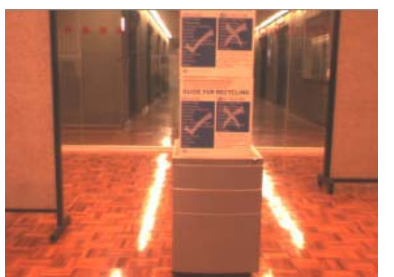

Fig. 10: Left image

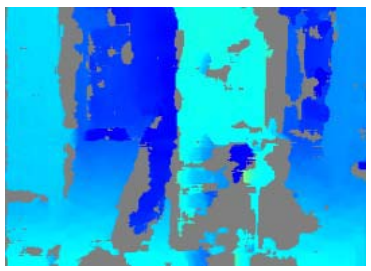

Fig. 12: Stereo disparity map

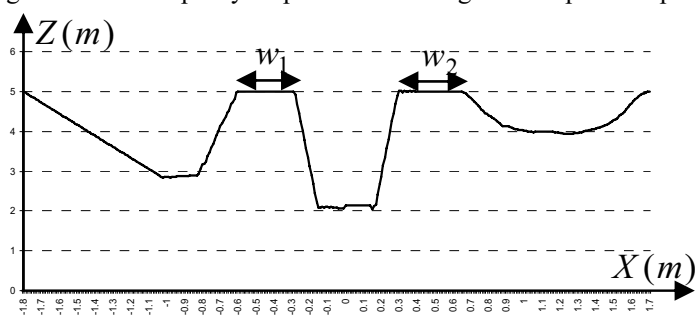

Fig. 14: 2D distance map

From the left and right images as shown in Fig. 10 and Fig. 11, the disparity and 3D point maps are shown in Fig. 12 and Fig. 13. From this 3D map, a 2D map with the widths of two freespaces $w_{1}, w_{2}$ is converted as shown in Fig. 14.

Assume that the actual widths of two freespaces $w_{a 1}$ and $w_{a 2}$ are equal to the safe diameter of the wheelchair $d_{s}$. This is difficult for the wheelchair to estimate and choose one of them to go through. For this reason, BR algorithm is used to find an optimal freespace and make the possible decision for the mobile wheelchair.

Consider the measured widths of two freespaces $w_{1}$ and $w_{2}$ as shown in Fig. 14. For similarity as Experiment 1, the high belief probabilities $P_{p o}\left(w_{1}\right), P_{p o}\left(w_{2}\right)$ and their average probability are shown in Table 2.

In this Table 2 , the average probability $P_{a v}\left(w_{1}\right)$, which is greater than not only the average probability $P_{a v}\left(w_{2}\right)$, but also the threshold probability $P_{t h}$, results in this freespace $w_{I}$ being selected for the wheelchair to move through.

Table 2: The estimated values of the widths of two freespaces

\begin{tabular}{|c|c|c|c|c|}
\hline Times & $w_{1}(t)$ & $P_{p o}\left(w_{1}(t)=F S\right)$ & $w_{2}(t)$ & $P_{p o}\left(w_{2}(t)=F S\right)$ \\
\hline 1 & 1010 & 0.7778 & 1020 & 0.7778 \\
\hline 2 & 1000 & 0.9617 & 1010 & 0.9617 \\
\hline 3 & 1020 & 0.9680 & 1020 & 0.9680 \\
\hline 4 & 980 & 0.7652 & 1000 & 0.9682 \\
\hline 5 & 1010 & 0.9613 & 1010 & 0.9682 \\
\hline 6 & 1020 & 0.9680 & 1010 & 0.9682 \\
\hline 7 & 1020 & 0.9682 & 1000 & 0.9682 \\
\hline 8 & 990 & 0.7652 & 990 & 0.7652 \\
\hline 9 & 1000 & 0.9613 & 980 & 0.7269 \\
\hline 10 & 1010 & 0.9680 & 1020 & 0.9600 \\
\hline \multicolumn{2}{|c|}{ Average } & $\mathbf{0 . 9 0 6 5}$ & & $\mathbf{0 . 9 0 3 2}$ \\
probability $P_{a v}$ & & & $\mathbf{0 . 0 1 0 4}$ \\
\hline \multicolumn{2}{|c|}{ Variance } & $\mathbf{0 . 0 0 9 0}$ & & \\
\hline
\end{tabular}

\section{CONCLUSION}

In this paper, stereoscopic vision, freespace detection in a 2D distance map and a BR algorithm are presented. The stereo disparity map is based on a combination of the left and right stereoscopic camera system images. From this disparity map, a 3D point map is computed based on a geometric projection approach. In addition, a 2D distance map is generated from this $3 \mathrm{D}$ map. Computation is performed on the 2D distance map to identify the presence of freespaces. For the freespace detection implementation for an autonomous wheelchair, a BR algorithm is applied to estimate the width of freespace. Experiment results on the wheelchair in a practical environment have illustrated the effectiveness of this estimation method.

\section{REFERENCES}

[1] R. C. Simpson, "Smart Wheelchairs: A Literature Review," Journal of Rehabilitation Research \& Dev, vol. 42, no 4, pp. 423-436, 2005.

[2] P. Saeedi, P. D. Lawrence, and D. G. Lowe, "Vision-Based 3-D Trajectory Tracking for Unknown Environments," IEEE Trans. on Robotics and Automation, vol. 22, no. 1, pp. 119-136, 2006.

[3] Y. Adachi, K. Goto, Y. Matsnmoto, and T. Ogasawara, "Development of Control Assistant System for Robotic Wheelchair Estimation of User's Behavior based on Measurements of Gaze and Environment," Proceedings of the IEEE on Computational Intelligence in Robotics and Automation, pp. 538-543, 2003.

[4] C. Watman, D. Austin, N. Barnest, G. Overett, and S. Thompson, "Fast Sum of Absolute Differences Visual Landmark Detector," Proceedings of the 2004 IEEE International Conference on Robotics 8 Automation, pp. 4827-4832, 2004.

[5] Thanh H. Nguyen, Jordan S. Nguyen, Duc M. Pham, and Hung T. Nguyen, "Real-Time Obstacle Detection for an Autonomous Wheelchair Using Stereoscopic Cameras," The 29th Annual International Conf. of the IEEE EMBC, pp. 4775 - 4778, 2007.

[6] G. N. Desouza and A. C. Kak, "Vision for Mobile Robot Navigation: A Survey," IEEE Transactions on Pattern Analysis and Machine Intelligence, vol. 24, no. 2, pp. 237-267, 2002.

[7] D. Murray and J. J. Little, "Using Real-Time Stereo Vision for Mobile Robot Navigation," Auto. Robots, vol. 8, pp. 161-171, 2000.

[8] Y. L. IP, A. B. Rad, and Y. K. Wong, "Autonomous Exploration and Mapping in An Unkown Environment," Proceedings of the IEEE Third International Conference on Machine Learning and Cybernetics, pp. 4194-4199, 2004

[9] V. Fox, J. Hightower, L. Liao, D. Schulz, and G. Borriello, "Bayesian Filtering for Location Estimation," IEEE Pervasive Computing, vol. 2, no. 3, 2003. 